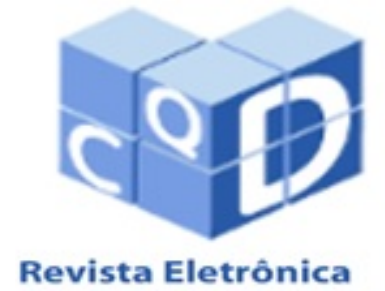

Revista Eletrônica

Paulista de Matemática

ISSN 2316-9664

Volume 11, dez. 2017

Edição Iniciação Científica

Elenice Weber Stiegelmeier

Universidade Tecnológica

Federal do Paraná

elenicew@utfpr.edu.br

\section{Anderson Inácio Salata de}

Abreu

Universidade Tecnológica

Federal do Paraná

andersinacio@hotmail.com

\section{Renata Toncovitch das Neves}

Universidade Tecnológica

Federal do Paraná

re_toncovitch@hotmail.com

\section{Dinâmica do crescimento de tumores com controle através do modelo linear quadrático}

Control of tumor growth by squared linear model

\begin{abstract}
Resumo
Neste trabalho será analisada a dinâmica de um modelo de crescimento tumoral com aplicação de controle através do modelo linear-quadrático para a radioterapia. O crescimento do tumor é modelado a partir das células normais, imunológicas e tumorais, representado por um sistema de equações diferenciais ordinárias. O objetivo principal é transferir o sistema de um regime com câncer para um ponto de equilíbrio estável livre de tumor através aplicação de controle por radioterapia. O efeito do tratamento por radioterapia será simulado pela aplicação do modelo linearquadrático. Com esta metodologia é possível analisar o efeito de protocolos de tratamento de radioterapia, além de efeitos relativos ao grau de severidade do tumor e à resistência do sistema imunológico do paciente. Simulações numéricas mostram o tratamento utilizando diversos protocolos presentes na literatura.

Palavras-chave: Sistema de equações diferencias ordinárias. Modelagem do crescimento tumoral. Modelo linear quadrático. Estabilidade.
\end{abstract}

\begin{abstract}
The dynamic of tumor growth model with control using the squared linear model for radiotherapy is analised. The tumor growth is modeled by ordinary cells, immunological cells and tumor cells, represented by a ordinary differential equations system. The main objective is move the system of a regime with cancer for a free stable equilibrium point of tumor by radiotherapy. The effect of radiotherapy treatment will be simulated by squared linear model. Using this methodology is possible to analyze the effects of treatment protocols by radiotherapy, also effects for the severity grade of the tumor and system immunological resistance of patient. $\mathrm{Nu}-$ merical simulations show the treatment using several protocols in literature.
\end{abstract}

Keywords: Ordinary differential equation system. Tumor growth modeling. Squared linear model. Equilibrium. 


\section{Introdução}

Apesar do grande avanço no diagnóstico e tratamento, o câncer ainda é um grande desafio para a comunidade científica. Nas últimas estatísticas sobre as tendências na incidência de câncer e mortalidade no mundo, verificou-se que os casos de câncer estão crescendo a um ritmo alarmante e ressalta-se a necessidade da aplicação urgente de estratégias de prevenção e tratamento eficazes para conter a doença [1].

Segundo Instituto Nacional do Câncer José de Alencar (INCA) o câncer é definido como o nome dado a um conjunto de mais de 100 doenças, que têm em comum o crescimento desordenado de células que invadem tecidos e órgãos. Essas células são conhecidas como cancerígenas e tendem a ser agressivas e incontroláveis [2].

O câncer, também conhecido como tumor, se classifica em duas classes, tumor benigno e tumor maligno. O tumor benigno é constituído por células semelhantes às de sua origem, seu crescimento é lento em grande parte dos casos, e pode ser totalmente removido cirurgicamente. O tumor maligno é formado pela mutação das células que o originaram sendo classificado como um tumor agressivo com alta capacidade de se infiltrar em outros órgãos e, ainda, suas células se multiplicam rapidamente. $\mathrm{O}$ tratamento e a possível cura depende de vários fatores, entre eles o diagnóstico precoce e a eficácia do tratamento [2].

As principais modalidades de tratamento para o câncer são a cirurgia, a imunoterapia, a quimioterapia e a radioterapia. A escolha do tratamento para o controle do câncer depende de alguns fatores, tais como: o estágio e a agressividade do tumor; o estado em que o sistema imunológico do paciente se encontra; o local onde está alojado o tumor. Assim, para auxiliar na tomada de decisão de qual tratamento utilizar, vários estudos estão sendo desenvolvidos com o auxílio da modelagem matemática com o objetivo de minimizar os efeitos colaterais causados pelo tratamento aos pacientes [3].

Uma estratégia para combater o crescimento desregulado das células tumorais é o tratamento por radioterapia, o qual emprega feixes de radiação ionizantes para reduzir as células tumorais. No entanto, a radiação pode danificar tanto as células normais quanto as células tumorais, devido à fase mitótica, ou seja, devido a duplicação celular. Nesta fase, as células se tornam mais vulneráveis a radiação e, ainda como as células tumorais apresentam um crescimento mais rápido que as células normais, a morte de células tumorais é maior comparado a morte de normais.

Na última década, vários autores têm apresentado estratégias de tratamento de câncer através da quimioterapia ou radioterapia [4-6]. Em [6] são descritas situações clínicas simuladas sobre o efeito da fração de tempo na modalidade de radioterapia intensiva modulada. O uso da radioterapia como tratamento seguem prescrições padrão que são obtidas a partir de observações empíricas. Segundo [3], isto acontece em parte pelo fracasso de formulações antigas e em parte por causa da falta de dados radiobiológicos precisos para pacientes individuais.

No modelo proposto por [4], os parâmetros foram encontrados de forma qualitativa, visando a estabilidade do sistema, sem levar em consideração nenhum tipo de tumor em particular. Porém, as leis de crescimento adotadas são semelhantes aos modelos de crescimento adotados por vários autores, e permitem uma visão bastante coerente do comportamento do tumor na dinâmica do sistema. Em [4] foi apresentado o modelo linear-quadrático (LQ) para simular o efeito biológico de doses únicas de radiação aplicadas, utilizando um número mínimo de parâmetros ajustáveis. 
No presente trabalho será apresentado um estudo do modelo de crescimento de tumores e o modelo linear quadrático usado para modelar a dose de radioterapia como descrito em [4]. O objetivo principal é transferir o sistema de um regime com câncer para um ponto de equilíbrio estável livre de tumor. E ainda, serão realizadas simulações numéricas da aplicação de controle por radioterapia seguindo os protocolos padrões de tratamento, com o objetivo de eliminar as células tumorais e preservar ao máximo as células normais. Com isso, auxiliar na tomada de decisão de qual a melhor estratégia a ser adotada que minimize os efeitos colaterais causados aos pacientes.

\section{Modelagem do crescimento tumoral}

Sejam $N, T$ e $I$ as células normais, tumorais e imunológicas, respectivamente. O modelo de crescimento tumoral é estabelecido a partir das seguintes hipóteses: as células normais e as células tumorais são modelados pela lei de crescimento logístico e competem pelos recursos disponíveis, onde define-se $r_{i}$ a taxa crescimento e $b_{i}$ a capacidade de sobrevivência, sendo $i=1$ os parâmetros associados ao tumor e $i=2$ os parâmetros associados às células sadias, enquanto que as células do sistema imunológico e as células de tumor competem da forma presa - predador.

Para a construção do modelo algumas condições devem ser satisfeitas:

(i) Considera-se que a fonte das células imunológicas está fora do sistema, assim, pode-se assumir uma taxa de afluência $s$ constante na ausência de tumor, ou seja, na ausência de qualquer tumor existe uma taxa fixa de entrada de células imunes $(0 \leq s \leq 0,5)$. Considerase $d_{1}$ sendo a taxa per capita de mortalidade na presença de qualquer tumor, resultando em longo prazo uma população $s / d_{1}$.

(ii) A resposta imunológica na presença de células tumorais é modelada pelo termo de crescimento não linear $\frac{\rho I T}{\chi+T} \operatorname{com} \chi$ e $\rho$ constantes positivas.

(iii) A iteração entre as células tumorais e imunológicas pode ocasionar na morte das células tumorais ou na inatividade das células imunológicas, representada pelas equações de competição dadas por:

$$
\begin{aligned}
\frac{d I}{d t} & =-c_{1} I T \\
\frac{d T}{d t} & =-c_{2} I T
\end{aligned}
$$

onde $c_{1}$ e $c_{2}$ são coeficientes de competição.

Levando-se em consideração as condições (i), (ii) e (iii), o modelo de crescimento tumoral é dado por um sistema de equações diferenciais ordinários descrito por [4]:

$$
\begin{aligned}
\dot{N} & =r_{2} N\left(1-b_{2} N\right)-c_{4} T N \\
\dot{T} & =r_{1} T\left(1-b_{1} T\right)-c_{3} T N-c_{2} I T \\
\dot{I} & =s+\frac{\rho I T}{\chi+T}-c_{1} I T-d_{1} I
\end{aligned}
$$


com $I \geq 0,15$, ou seja, o número de células no organismo não será inferior a $0,15 \times 10^{11}$. A mesma escala vale para as células normais e tumorais. Note que o sistema (3)-(5) representa o crescimento de células tumorais, imunológicas e normais, sem qualquer intervenção de tratamento.

A Figura 1 ilustra as trajetórias do sistema tumoral sem aplicação de controle (tratamento). Note que as células tumorais a partir de 50 dias dominam o sistema, enquanto que as células normais e imunológicas diminuem. Logo, esta situação ilustra a importância do uso de algum tratamento para controlar a doença.

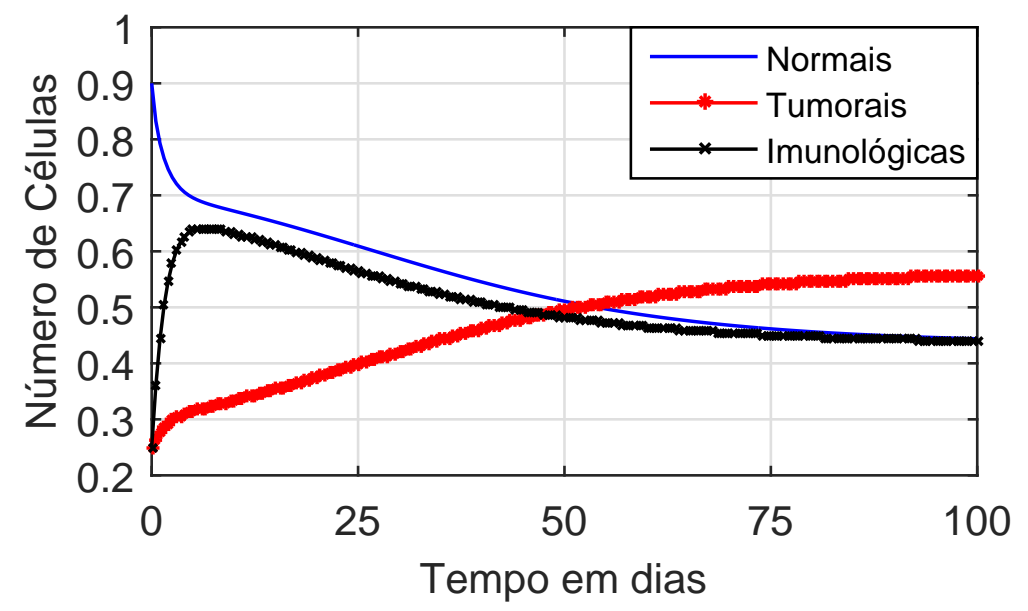

Figura 1: Dinâmica do sistema tumoral sem aplicação de controle.

\section{Análise de equilíbrio}

Considere o sistema (3)-(5) sem aplicação de controle. O paciente será considerado curado, quando o sistema esta em torno de uma bacia de atração estável livre de células tumorais, ou então, em torno de uma bacia de atração estável na qual só exista uma pequena quantia de células tumorais.

Neste caso, tem-se três planos do sistema (3)-(5) sem a aplicação de droga, descritos por:

- $\tilde{N}_{N}$

$$
\dot{N}=0 \Rightarrow\left\{\begin{array}{l}
N=0 \\
N=\frac{1}{b_{2}}-\left(\frac{c_{4}}{b_{2} r_{2}}\right) T .
\end{array}\right.
$$

$\tilde{N}_{N}$ é a união de dois planos, $I-T$ são as coordenadas do plano e $N_{N}$ é o plano paralelo ao eixo-I.

Seja $g(T)$ a função que descreve $N_{N}$ em termos da população tumoral:

$$
g(T)=\frac{1}{b_{2}}-\left(\frac{c_{4}}{b_{2} r_{2}}\right) T
$$


- $\tilde{N}_{T}$

$$
\dot{T}=0 \Rightarrow\left\{\begin{array}{l}
T=0 \\
T=\frac{1}{b_{1}}-\left(\frac{c_{2}}{b_{1} r_{1}}\right) I-\left(\frac{c_{3}}{b_{1} r_{1}}\right) N
\end{array}\right.
$$

- $\tilde{N}_{I}$

$$
\dot{I}=0 \Rightarrow I=\frac{s(\chi+T)}{\left(c_{1} T-d_{1}\right)(\chi+T)-\rho T}
$$

com $\rho T \neq\left(c_{1} T-d_{1}\right)(\chi+T)$ e $N_{I}$ uma superfície cilíndrica encurvada paralela ao eixo-N.

Considera-se $f(T)$ a função da população tumoral que descreve $N_{I}$ dada por:

$$
f(T)=\frac{s(\chi+T)}{\left(c_{1} T-d_{1}\right)(\chi+T)-\rho T} .
$$

Através dessas análises, pode-se classificar os pontos de equilíbrio de acordo com os pontos de intersecções das superfícies. Maiores detalhes sobre a análise de equilíbrio são descritos em [4].

Os pontos de equilíbrio do sistema de crescimento tumoral, sem aplicação de controle, são classificados em livre de tumor, morto e de coexistência.

1. Livre de tumor. Considere a população de células tumorais como sendo zero. No entanto, as células normais sobrevivem, ou seja,

$$
\begin{aligned}
N & =r_{2} N\left(1-b_{2} N\right) \\
T & =0 \\
I & =s-d_{1} I .
\end{aligned}
$$

Assim, o ponto de equilíbrio é $(N, T, I)=\left(\frac{1}{b_{2}}, 0, \frac{s}{d_{1}}\right)$.

2. Morto. Considere a população de células normais sendo zero. Então, tem-se dois possíveis pontos de equilíbrio:

- Tipo 1: Células normais e tumorais sendo nulas.

$$
\begin{aligned}
N & =0 \\
T & =0 \\
I & =s-d_{1} I .
\end{aligned}
$$

Logo, o ponto de equilíbrio é $(N, T, I)=\left(0,0, \frac{s}{d_{1}}\right)$.

- Tipo 2: Células normais sendo nulas e células tumorais sobrevivem. Assim, o ponto de equilíbrio será $(N, T, I)=(0, a, f(a))$.

Considera-se $a$ uma solução não-negativa de:

$$
a+\left(\frac{c_{2}}{r_{1} b_{1}}\right) f(a)-\frac{1}{b_{1}}=0
$$


onde $f(a)$ é obtida através da população de células tumorais e representa a população de células imunológicas dada por $(T=a)$ :

$$
f(a)=\frac{s(\alpha+T)}{c_{1} T(\alpha+T)+d_{1}(\alpha+T)-\rho T} .
$$

3. Coexistência. Considere as populações tumorais e normais sendo diferente de zero e coexistem. Então, o ponto de equilíbrio é dado por $(N, T, I)=(g(b), b, f(b))$. Considera-se $b$ uma solução não-negativa de:

$$
b+\left(\frac{c_{2}}{r_{1} b_{1}}\right) f(b)+\left(\frac{c_{3}}{r_{1} b_{1}}\right) g(b)-\frac{1}{b_{1}}=0,
$$

onde $g(b)$ é obtida através da população de células tumorais e representa a população de células normais dada por $(T=b)$ :

$$
g(b)=\frac{1}{b_{2}}-\left(\frac{c_{4}}{c_{2}}\right) T .
$$

\subsection{Ponto de equilíbrio livre do tumor}

Nessa seção será analisado o ponto de equilíbrio do sistema (3)-(5) livre de tumor, uma vez que o objetivo é eliminar o tumor do organismo, e ainda, será determinado os parâmetros para que o ponto de equilíbrio seja localmente estável.

A matriz Jacobiana correspondente ao sistema (3)-(5) é dada por:

$$
J=\left[\begin{array}{ccc}
r_{2}\left(1-2 b_{2} N\right)-c_{4} T & -c_{4} N & 0 \\
-c_{3} T & r_{1}\left(1-2 b_{1} T\right)-c_{2} I-d_{1}-c_{3} N & -c_{2} T \\
0 & \frac{\rho \chi I}{(\chi+T)^{2}}-c_{1} I & -c_{1} T-d_{1}
\end{array}\right] .
$$

A partir da matriz Jacobiana $J$, avaliando-se a linearidade no ponto $P=\left(\frac{1}{b_{2}}, 0, \frac{s}{d_{1}}\right)$ obtêm-se os seguintes autovalores:

$$
\begin{gathered}
\lambda_{1}=-r_{2}, \\
\lambda_{2}=r_{1}-\frac{c_{3}}{b_{2}}-\frac{c_{2} s}{d_{1}}, \\
\lambda_{3}=-d_{1} .
\end{gathered}
$$

Portanto, o sistema é estável no ponto $P$ quando $\lambda_{1}, \lambda_{2}$ e $\lambda_{3}$ são negativos. Como $-r_{2}<0$, tem-se $\lambda_{1}<0$ e, ainda, $-d_{1}<0$, então, $\lambda_{3}<0$.

Analisando o caso em que $\lambda_{2}$ é negativo, tem-se:

$$
\lambda_{2}<0 \Rightarrow r_{1}<\frac{c_{1} s}{d_{1}}+\frac{c_{3}}{b_{2}} .
$$

A expressão (6) mostra que se o ponto de equilíbrio é instável, então, de acordo com o modelo (3)-(5) nenhum tratamento será capaz de controlar o tumor.

A Figura 2 ilustra o comportamento das células normais e imunológicas sem a presença das células tumorais. Observe que o sistema têm comportamento logístico, isto é, existe um limitante para o crescimento das células. 


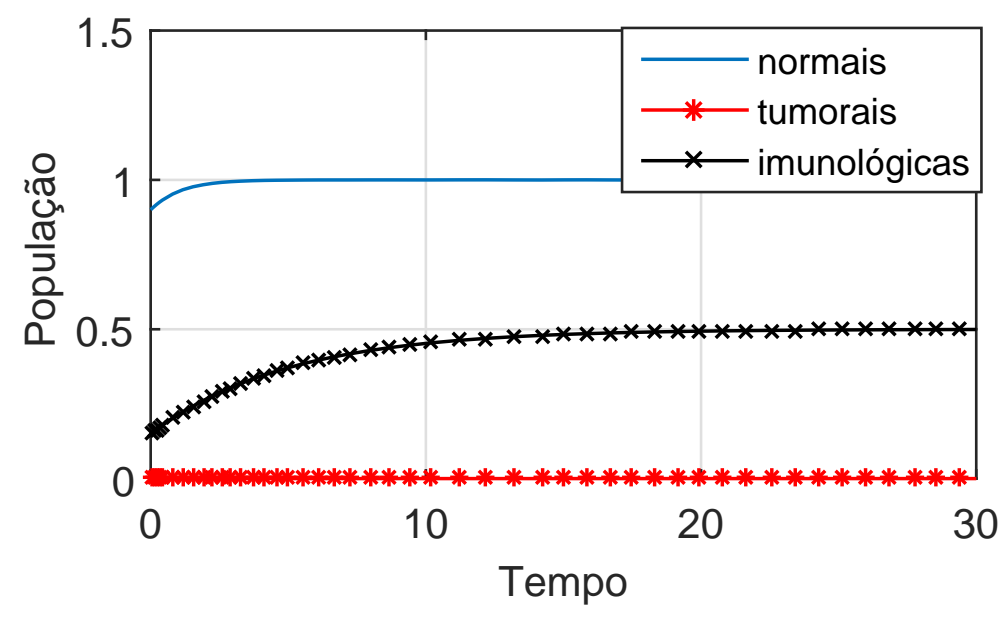

Figura 2: Dinâmica do sistema tumoral sem a presença de células tumorais.

\section{Radioterapia e o modelo linear quadrático}

O modelo Linear Quadrático (LQ) é amplamente utilizado para modelar as doses aplicadas de radiologia, tendo como objetivo avaliar a fração de morte celular radio-induzida. Esse modelo foi desenvolvido por Douglas e Fowler [7] com o objetivo de descrever a relação entre a sobrevivência das células e a dosagem absorvida em um sistema.

A fração sobrevivente para este tipo de situação, pode ser descrita por:

$$
S=e^{-n \alpha d-n \beta d^{2}}
$$

onde, $S$ fração de células sobreviventes, $n$ número de aplicações, $d$ dose por fração, $\alpha$ número de células mortas por por $G y$ (unidade de medida de dose absorvida), $\beta$ número de células mortas por $(G y)^{2}$ em escala logarítmica do componente quadrático. O comportamento do modelo linear quadrático é descrito na Figura 3.

O modelo LQ é usado para simular as doses de radioterapia aplicadas. A radioterapia consiste em um tratamento que através de feixes de radiação ionizantes possibilita a destruição de células, principalmente das células tumorais. Observe que as radiações ionizantes são carregadas de energia, assim, ao entrar em contato com algum tecido do corpo dão origem a eletróns que geram efeitos químicos como, por exemplo, a hidrólise da água e a ruptura das cadeias de ADN, com isso, a morte das células ocorre por vário fatores [2].

A dosagem do tratamento é estipulada segundo alguns protocolos de tratamento e o tempo de aplicação varia conforme a especificidade de cada indivíduo e do tumor. Porém, todas as aplicações tem o mesmo objetivo, diminuir ou exterminar as células tumorais causando o menor dano possível para células normais presentes no tecido analisado. Quanto mais rápido o tumor diminuir, mais radiossensível o tumor se torna. A radiossensibilidade está diretamente ligada à origem da célula, a oxigenação e a forma clínica da apresentação do tumor, grande parte dos tumores radiossensíveis são radiocuráveis. Para que o efeito da radioterapia atinja o maior número de células neoplásicas e seja respeitada a matança de células normais, a dose total do tratamento é administrada normalmente fracionada em doses diárias iguais [1]. 
O tratamento por radioterapia pode ser prescrita como único ou associada à outros métodos terapêuticos tais como quimioterapia, imunoterapia, etc. Além de poder ser combinada a cirurgia, com a aplicação, pré-operatória ou pós-operatória. Os efeitos colaterais causados pelo radioterapia são normalmente tolerados pelo paciente, desde que os princípios de matança de células normais e o fracionamento da dose total seja atendido [2].

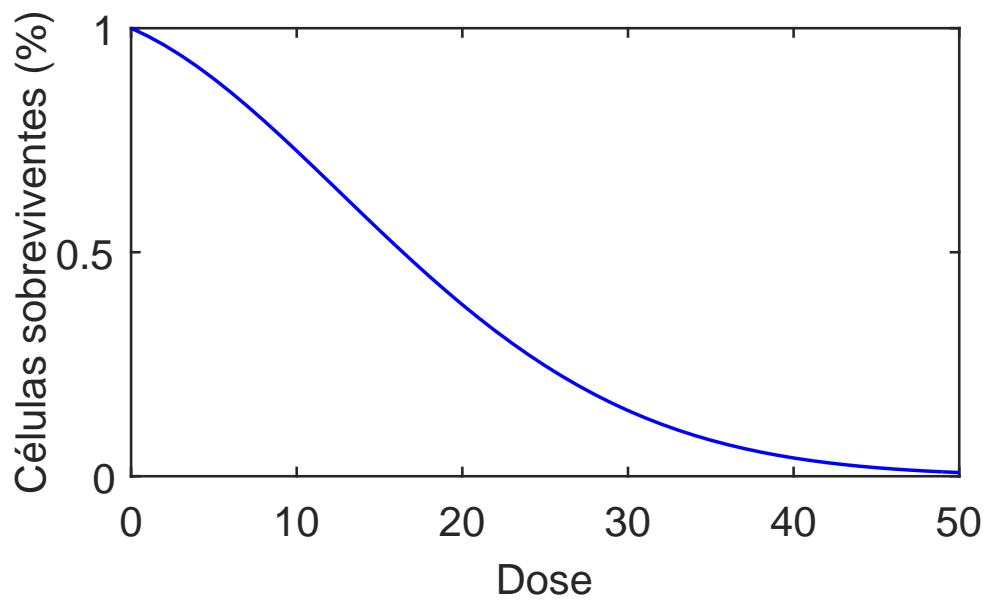

Figura 3: Dinâmica do modelo Linear Quadrático.

\section{Simulações numéricas}

Para as simulações numéricas é conveniente tratar o problema de controle de crescimento tumoral de forma discretizada, uma vez que o câncer evolui com o tempo $t$. Dessa forma, considere o modelo de crescimento tumoral discreto dado da seguinte forma:

$$
\begin{aligned}
N_{t+1} & =r_{2} N_{t}\left(1-b_{2} N_{t}\right)-c_{4} T_{t} N_{t} \\
T_{t+1} & =r_{1} T_{t}\left(1-b_{1} T_{t}\right)-c_{3} T_{t} N_{t}-c_{2} I_{t} T_{t} \\
I_{t+1} & =s+\frac{\rho I_{t} T_{t}}{\chi+T_{t}}-c_{1} I_{t} T_{t}-d_{1} I_{t} .
\end{aligned}
$$

A fração de morte celular induzida pela aplicação da radioterapia no controle das células tumorais é uma função da dose, $d(G y)$, aplicada no local do tumor e é dada pela curva doseresposta [7]:

$$
F_{i}\left(d_{t}\right)=a_{i}\left(1-e^{n \alpha_{i} d-n \beta_{i} d^{2}}\right)
$$

sendo $F_{i}$ a fração de células mortas a cada dose aplicada no local do tumor, $d_{t}$ dose por fração no tempo $t, n$ o número de aplicações da dose de radioterapia, $i=1,2,3$ indicam os parâmetros relacionados as células tumorais, normais e imunológicas, respectivamente. 
Tabela 1: Protocolos padrão de tratamento por radioterapia (dados extraídos de [7] e [8]).

\begin{tabular}{ccccc}
\hline Protocolos & $\begin{array}{c}\text { Tecido de } \\
\text { Resposta }\end{array}$ & $\begin{array}{c}\text { Tempo de } \\
\text { Tratamento }\end{array}$ & $\begin{array}{c}\text { Dose } \\
\text { Total }(\mathbf{G y})\end{array}$ & $\begin{array}{c}\text { Indicado para } \\
\text { Tumores }\end{array}$ \\
\hline 01 & Precoce & $2-3$ semanas & 30,00 & Cabeça e Pescoço \\
02 & Precoce & $6-7$ semanas & 60,00 & Mama \\
03 & Precoce & $0-1$ semanas & 20,00 & Melenoma Ocular \\
04 & Precoce & $3-4$ semanas & 40,00 & Pele(eritema) \\
05 & Precoce & $5-6$ semanas & 30,00 & Pulmão \\
06 & Tardia & $6-7$ semanas & 66,00 & Próstata \\
\hline
\end{tabular}

Incluindo ao sistema (8)-(10), o controle dado pelo modelo linear quadrático (11), para simular o tratamento por radioterapia, obtêm-se:

$$
\begin{aligned}
N_{t+1} & =r_{2} N_{t}\left(1-b_{2} N_{t}\right)-c_{4} T_{t} N_{t}-\left(1-F_{i}\right) N_{t} \\
T_{t+1} & =r_{1} T_{t}\left(1-b_{1} T_{t}\right)-c_{3} T_{t} N_{t}-c_{2} I_{t} T_{t}-\left(1-F_{i}\right) T_{t} \\
I_{t+1} & =s+\frac{\rho I_{t} T_{t}}{\chi+T_{t}}-c_{1} I_{t} T_{t}-d_{1} I_{t}-\left(1-F_{i}\right) I_{t} .
\end{aligned}
$$

com $F_{i}\left(d_{t}\right)$ fração de células mortas no tempo $t$.

A fração células mortas, $F_{i}$ é considerado como um evento externo ao sistema e atua na forma de "sinais impulsivos", ou seja, é uma grandeza absoluta que afeta o crescimento das células visando a eliminação do tumor. Considera-se que a fração $F_{i}$ está relacionada com o passo temporal, então, esta forma de controle do sistema (8)-(10) pode ser contínua (sem interrupção do tratamento nos finais de semana) ou descontínua (com interrupção do tratamento nos finais de semana) dependendo de cada protocolo de tratamento. O tamanho da dose por fração e o número de aplicações são determinados segundo os protocolos de tratamento padrão por radioterapia descritos na Tabela 1

De acordo com os estudos realizados por [5], o objetivo do tratamento por radioterapia é a eliminação do tumor, afetando o mínimo possível as células normais. Para tanto, visando diminuir as complicações tardias para os tecidos normais e manter o número de células normais em níveis mínimos de sobrevivência, foram adotados os seguintes critérios:

(i) A cada aplicação de radioterapia a fração de morte celular das células normais $\left(F_{n}\right)$ não pode ser superior à $44 \%$ do estado inicial, ou seja, no tempo $t+1$ a população de células deve ser maior ou igual $66 \%$ em relação ao tempo anterior $t$.

(ii) A população de células normais não deve ser inferior a 0,44 unidades normalizadas do estado considerado saudável $\left(\frac{1}{b_{2}}\right)$.

A Figura 4 ilustra a estratégia de controle do tumor através do tratamento por radioterapia. Os parâmetros de entrada são: o número de células iniciais (condições iniciais do sistema) e o protocolo de tratamento considerado de acordo com o diagnóstico da doença. Neste diagrama, as variáveis do sistema de crescimento tumoral (N, T e I) são representadas por y e o modelo 


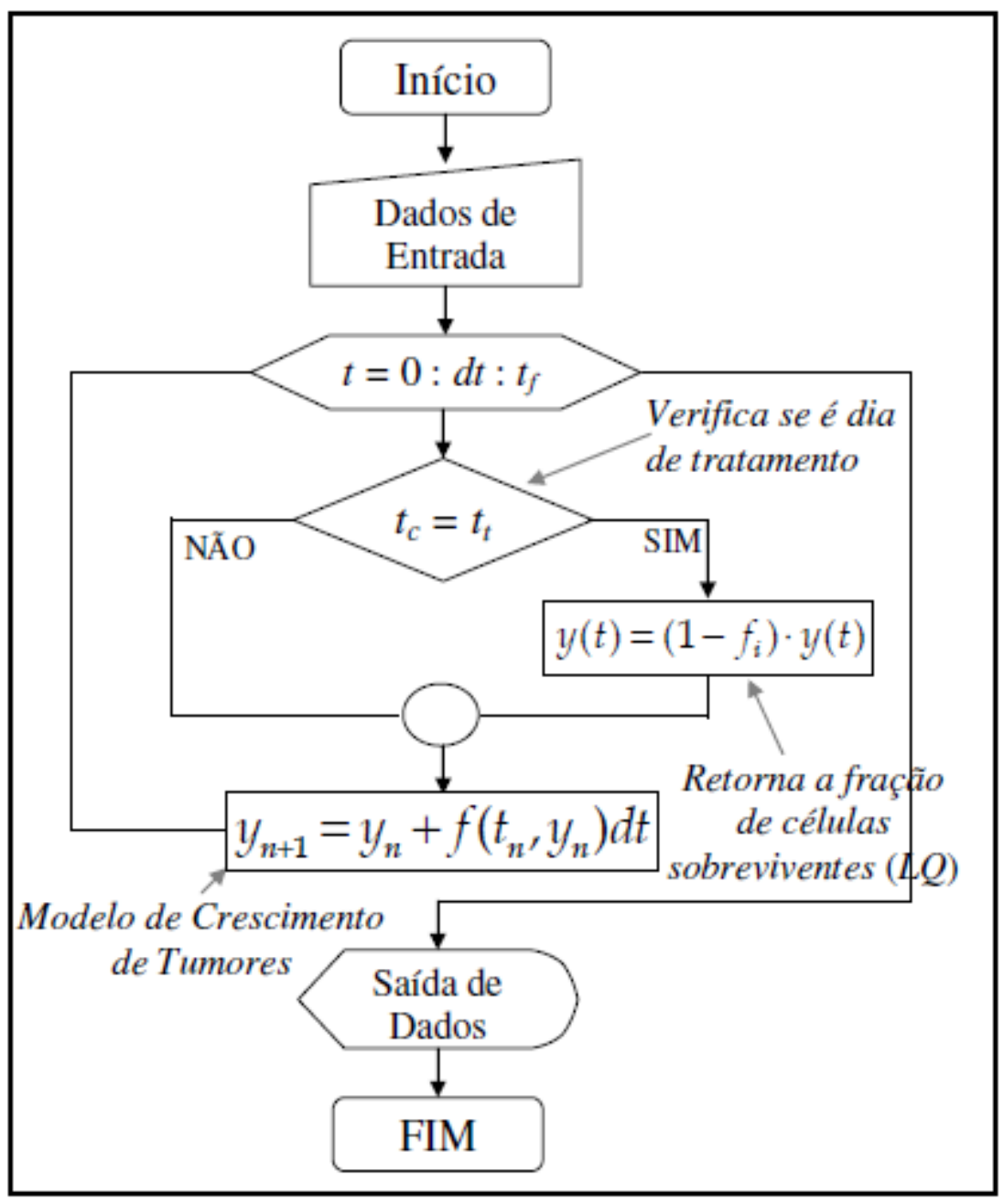

Figura 4: Fluxograma da estratégia de controle por radioterapia.

dinâmico do sistema tumoral é resolvido pelo Método de Runge Kutta de $4^{\mathrm{a}}$. ordem com o auxilio do software Matlab.

Para as simulações numéricas foram utilizados os protocolos apresentados na Tabela 1 e com as condições iniciais $N_{0}=0,9, T_{0}=0,25$ e $I_{0}=0,15$. Os demais parâmetros do sistema tumoral foram extraídos de [4] e estão descritos a seguir:

$$
\begin{array}{lllll}
a_{1}=0,2 & a_{2}=0,3 & a_{3}=0,1 & b_{1}=1,0 & b_{2}=1,0 \\
\alpha=0,3 & c_{1}=1,0 & c_{2}=0,5 & c_{3}=1,0 & c_{4}=1,0 \\
d_{1}=0,2 & r_{1}=1,5 & r_{2}=1,0 & s=0,33 & \rho=0,01
\end{array}
$$

Note que no caso do equilíbrio livre de tumor, $(T=0)$, o sistema é estável se o autovalor $\lambda_{2}$ correspondente for negativo:

$$
\lambda_{2}<0 \Rightarrow r_{1}<\frac{c_{1} s}{d_{1}}+\frac{c_{3}}{b_{2}}
$$


Considerando a inequação (15) e $r_{1}=2,66$, tem-se:

$$
2,66>\frac{0,33}{0,2}+1 \Longrightarrow 2,66>2,65 \text {. }
$$

Nesse caso, como o autovalor é positivo, o ponto de equilíbrio livre de tumor se torna instável, ou seja, de acordo com (15) nenhum tratamento será capaz de controlar o crescimento do tumor.

Para mostrar a influência do parâmetro $r_{1}$ na dinâmica tumoral são apresentados os estudos de caso descritos a seguir.

1. Estudo de caso: Melenoma Ocular.

Para o caso do Melenoma Ocular considera-se o tratamento convencional com $\frac{\alpha}{\beta}=10 G y$. De acordo com a Tabela 1 a dose total é de no máximo 20Gy, dividida em 10 aplicações de 2,0Gy durante 10 dias e $r_{1}=2.66$.

A Figura 5 ilustra o comportamento do sistema tumoral com tratamento convencional. Observe que mesmo com a dosagem máxima permitida (veja Tabela 1) a partir do $2^{\circ}$ dia de tratamento o tumor toma conta do sistema, levando o paciente ao óbito.

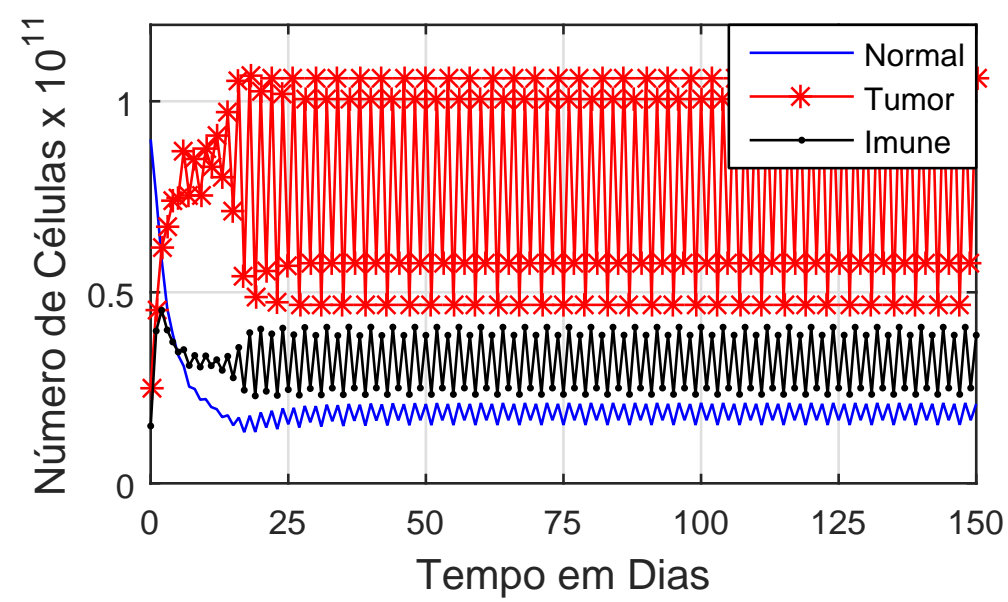

Figura 5: Tratamento convencional do melenoma ocular com dose total de $20 G y$ durante 20 dias.

2. Estudo de caso: Câncer de Pulmão.

No caso do tratamento para o câncer de pulmão, considera-se $\frac{\alpha}{\beta}=10 G y$. De acordo com a Tabela 1 a dose total é de no máximo 30Gy, dividida em 17 aplicações de 1,8Gy durante 17 dias.

A Figura 6 ilustra o comportamento do sistema tumoral com tratamento convencional. Note que mesmo com a dosagem máxima permitida (veja Tabela 1) a partir do $3^{\circ}$ dia de tratamento o tumor toma conta do sistema, assim posteriormente levando o paciente ao óbito. 


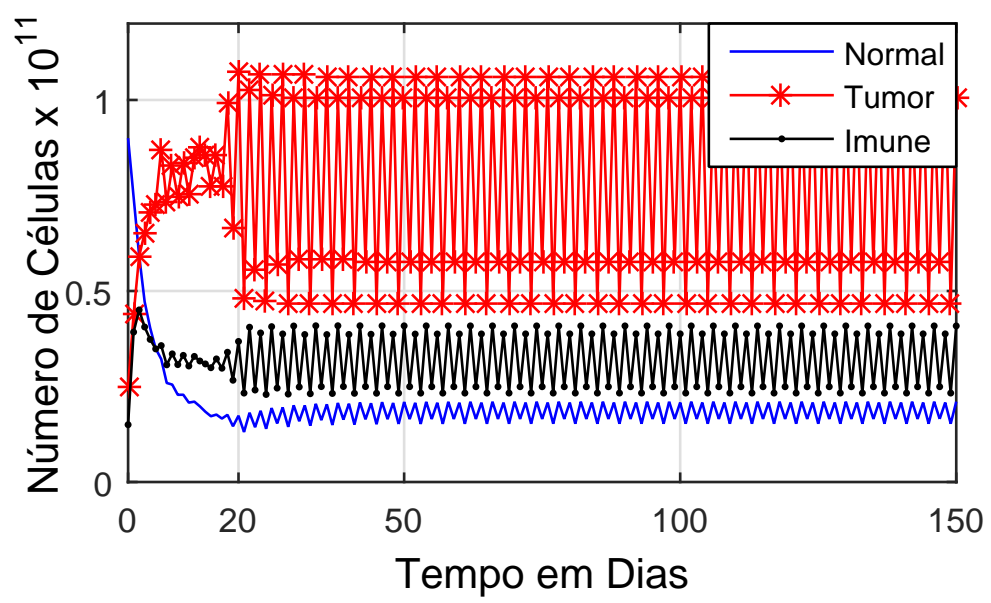

Figura 6: Tratamento convencional para o câncer de pulmão com dose total de 30 Gy durante 15 dias.

Tabela 2: Câncer de próstata

\begin{tabular}{ccccccc}
\hline $\begin{array}{c}\text { Tipo de } \\
\text { Fracionamento }\end{array}$ & $\begin{array}{c}\text { Tempo de } \\
\text { Eliminação do } \\
\text { do tumor }\end{array}$ & Dose/Fração & $\begin{array}{c}\text { Tempo de } \\
\text { Tratamento }\end{array}$ & $\begin{array}{c}\text { Dose } \\
\text { Total } \\
(\mathbf{G y})\end{array}$ & FN & FT \\
\hline Convencional & 56 dias & 37 frações de $1.8 \mathrm{~Gy}$ & 37 dias & 66.00 & 0.09 & 0.22 \\
Hipofracionamento & 48 dias & 20 frações de 3.3 Gy & 20 dias & 66.00 & 0.10 & 0.23 \\
\hline
\end{tabular}

\subsection{Resultados}

A seguir serão apresentados os resultados de simulações numéricas para casos específicos de cânceres afim de simular o tratamento por radioterapia e analisar a dinâmica do sistema de crescimento tumoral, considerando o parâmetro $r_{1}=1,5$. As simulações numéricas foram implementadas com o auxilio do sofware Matlab.

\subsubsection{Câncer de próstata}

Considere $\alpha / \beta=3 G y$ e utilizando o tratamento convencional e o hipofracionamento como forma de tratamento. O hipofracionamento consiste em doses diárias maiores do que o tratamento convencional, portanto, parece ser uma opção terapêutica interessante para este tumor, tendo em vista a possibilidade de melhora do índice terapêutico com uma alteração na dose diária de radioterapia [9]. Os resultados das simulações encontram-se na Tabela 2, com $F N$ a fração de células normais mortas por dose aplicada e $F T$ a fração de células tumorais mortas por dose aplicada.

De acordo com a Tabela 2 o tumor é eliminado do sistema após 48 dias utilizando o hipofraci- 


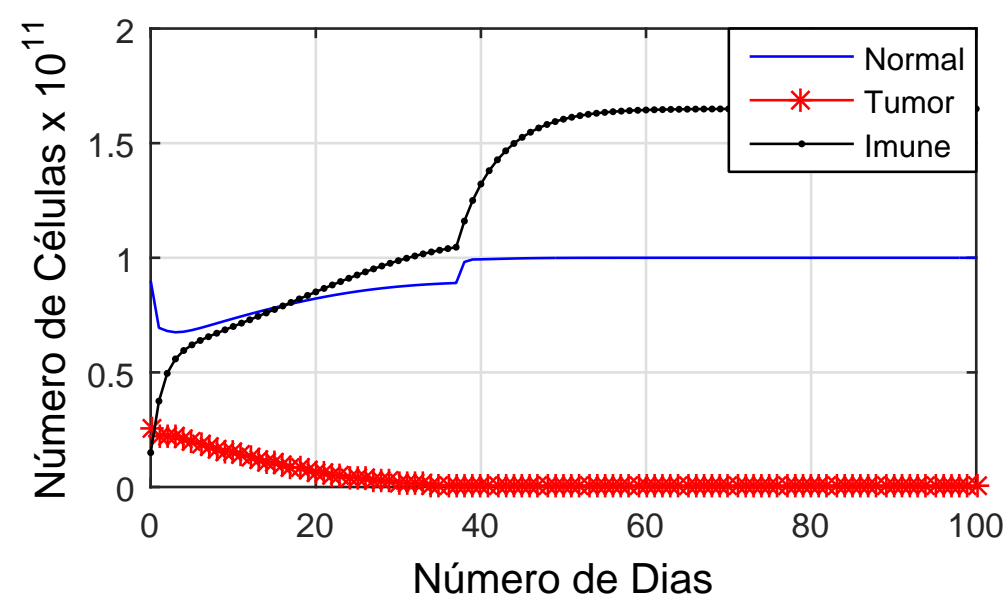

Figura 7: Simulação para o câncer de próstata utilizando o tratamento convencional.

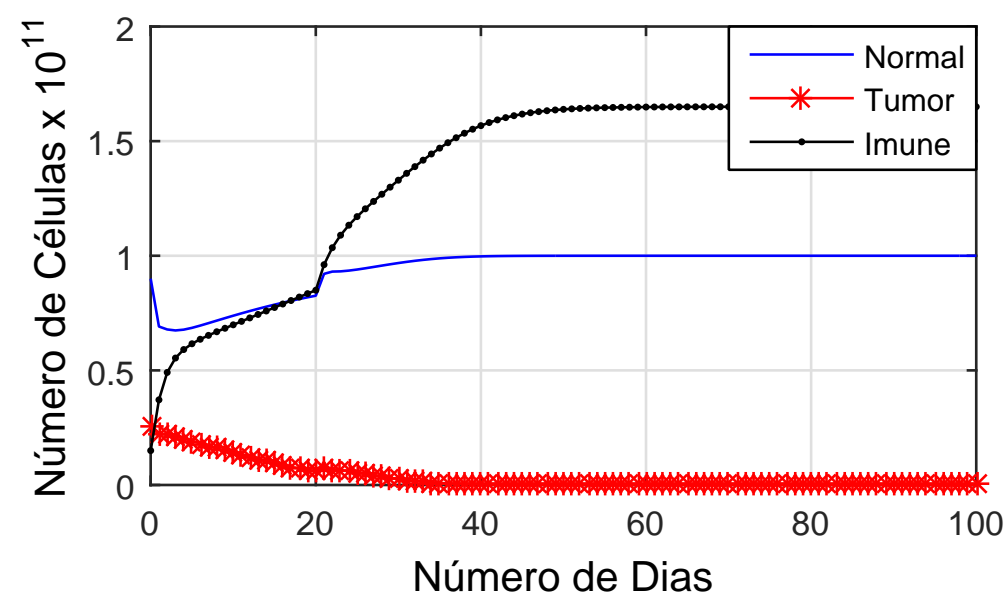

Figura 8: Simulação para o câncer de próstata utilizando o hipofracionamento.

onamento e 56 dias utilizando o tratamento convencional. Em ambos os tratamentos o paciente é curado. Assim, a partir dessas simulações o uso do hipofracionamento é mais indicado, visto que o tempo de tratamento e de eliminação do tumor é menor em comparação ao uso do tratamento convencional, conforme ilustrado nas Figuras 7 e 8.

\subsubsection{Câncer de cabeça e pescoço}

Considera-se $\alpha / \beta=10 G y$ e para as simulações foram utilizados o tratamento convencional e o hiperfracionamento. O hiperfracionamento consiste na divisão da dose convencional em duas frações diárias. A terapia com hiperfracionamento tem relatado significativa melhora no controle local e regional deste tipo de tumor [10]. Os resultados das simulações encontram-se na Tabela 3.

De acordo com a Tabela 3 o tumor é eliminado após 52 dias utilizando o tratamento conven- 
Tabela 3: Câncer de cabeça e pescoço

\begin{tabular}{cccccccc}
\hline $\begin{array}{c}\text { Tipo de } \\
\text { Fracionamento }\end{array}$ & $\begin{array}{c}\text { Tempo de } \\
\text { Eliminação do } \\
\text { do tumor }\end{array}$ & Dose/Fração & $\begin{array}{c}\text { Tempo de } \\
\text { Tratamento }\end{array}$ & $\begin{array}{c}\text { Dose } \\
\text { Total } \\
\text { (Gy) }\end{array}$ & FN & FT \\
\hline Convencional & 52 dias & 15 frações de $2.0 \mathrm{~Gy}$ & 15 dias & 30.00 & 0.03 & 0.12 \\
Hiperfracionamento & 30 dias & 30 frações de 1.0 Gy & 15 dias & 30.00 & 0.2 & 0.13 \\
\hline
\end{tabular}

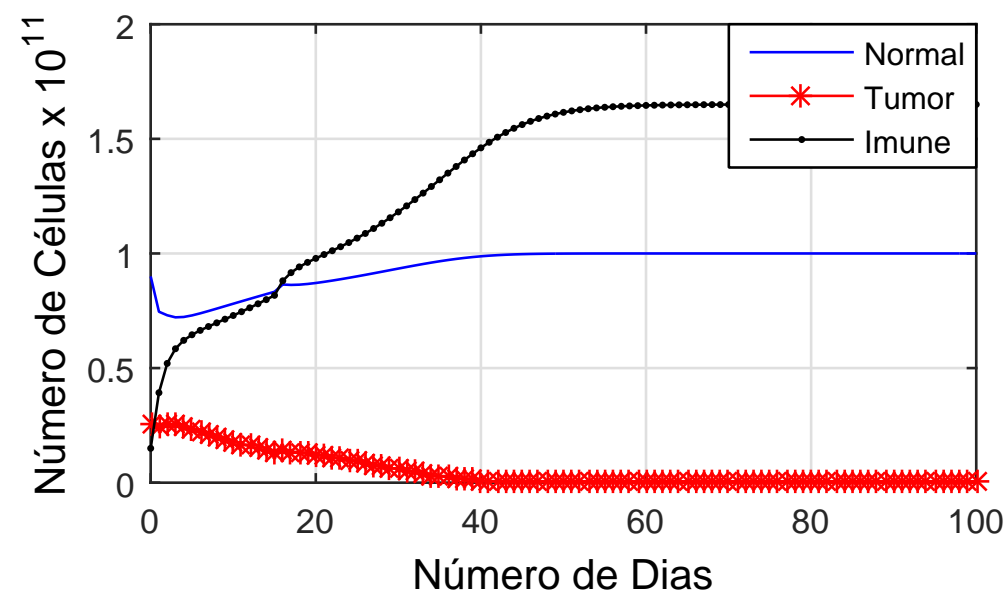

Figura 9: Simulação para o câncer de cabeça e pescoço utilizando o tratamento convencional.

cional e 30 dias utilizando o hiperfracionamento. O tempo de eliminação do tumor utilizando o hiperfracionamento é bem inferior comparado a utilização do convencional, assim, a partir dessa simulação numérica o tratamento com hiperfracionamento seria o mais indicado para este caso. Em ambos os casos o paciente é curado. As Figuras 9 e 10 ilustram ambos os tratamentos.

\subsubsection{Câncer de pele}

Considera-se $\alpha / \beta=10 G y$ e para as simulações foram utilizados os tratamentos convencional e o fracionamento acelerado. O fracionamento acelerado divide a dose convencional em três frações diárias. A escolha desse fracionamento baseia-se no estudos realizados por [3], que a partir de simulações numéricas obteve o melhor resultado para este tipo de câncer usando as mesmas condições aqui explicitadas. Os resultados das simulações encontram-se na Tabela 4.

De acordo com a Tabela 4 com o tratamento convencional o tumor é eliminado após 35 dias e 21 dias com o fracionamento acelerado. O tempo de eliminação do tumor utilizando o fracionamento acelerado é mais satisfatório, ainda que o FT seja menor, isto acontece pois o número de aplicações da radioterapia é 3 vezes maior do que o tratamento convencional. O paciente é curado em ambos os tratamentos. As Figuras 11 e 12 ilustram ambos os tratamentos. 


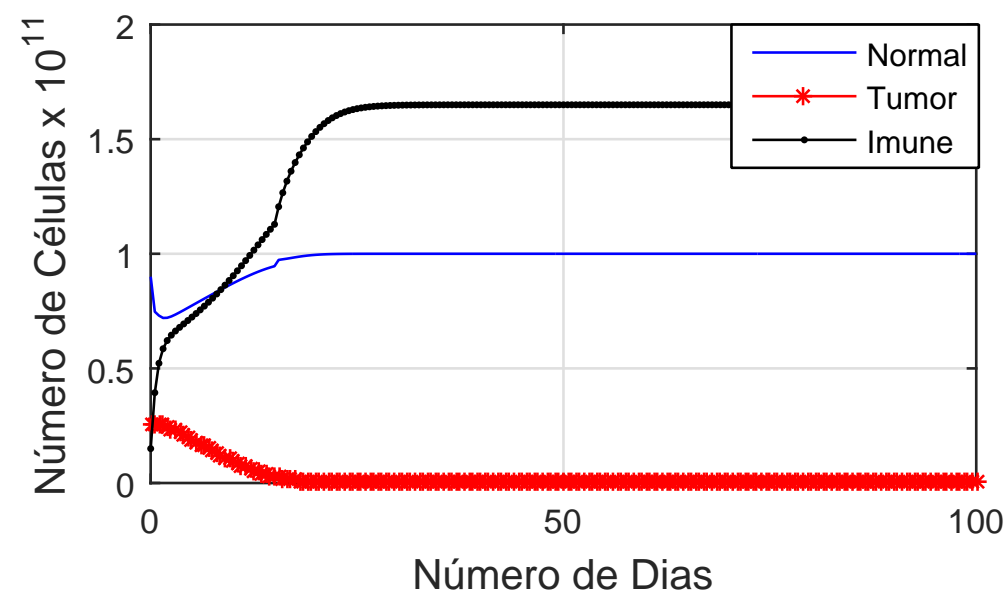

Figura 10: Simulação apara o câncer de cabeça e pescoço utilizando o hiperfracionamento.

Tabela 4: Câncer de pele

\begin{tabular}{ccccccc}
\hline $\begin{array}{c}\text { Tipo de } \\
\text { Fracionamento }\end{array}$ & $\begin{array}{c}\text { Tempo de } \\
\text { Eliminação do } \\
\text { do tumor }\end{array}$ & Dose/Fração & $\begin{array}{c}\text { Tempo de } \\
\text { Tratamento }\end{array}$ & $\begin{array}{c}\text { Dose } \\
\text { Total } \\
(\mathbf{G y})\end{array}$ & FN & FT \\
\hline Convencional & 35 dias & 25 frações de $1.6 \mathrm{~Gy}$ & 25 dias & 40.00 & 0.03 & 0.16 \\
F. Acelerado & 21 dias & 60 frações de $0.66 \mathrm{~Gy}$ & 20 dias & 30.00 & 0.03 & 0.14 \\
\hline
\end{tabular}

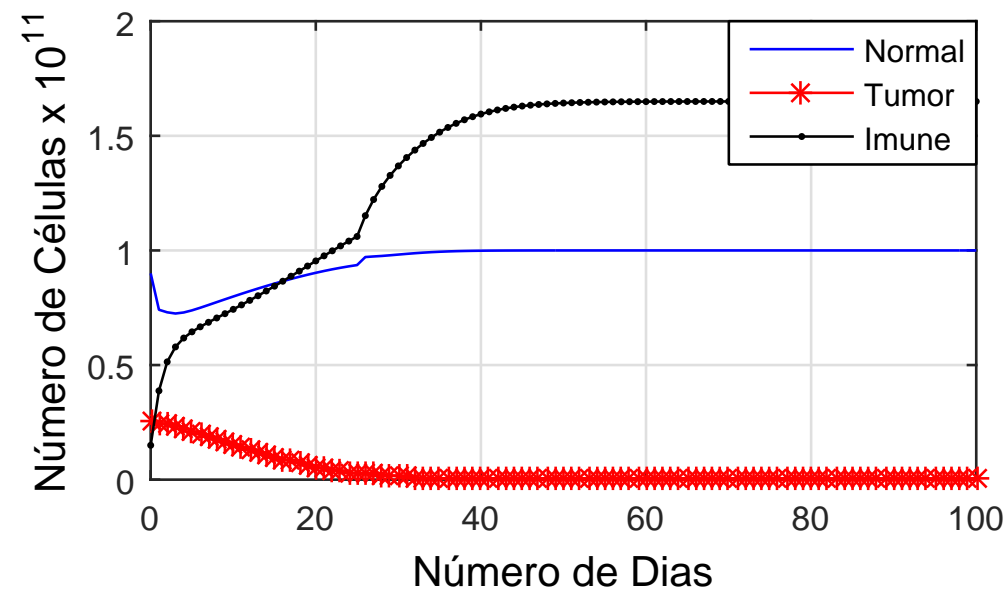

Figura 11: Simulação para o câncer de pele utilizando o tratamento convencional.

\subsubsection{Câncer de mama}

Neste caso, considera-se $\alpha / \beta=10 G y$ e para as simulações foram considerados os tratamentos convencional e o hipofracionamento. A utilização do hipofracionamento para o câncer de 


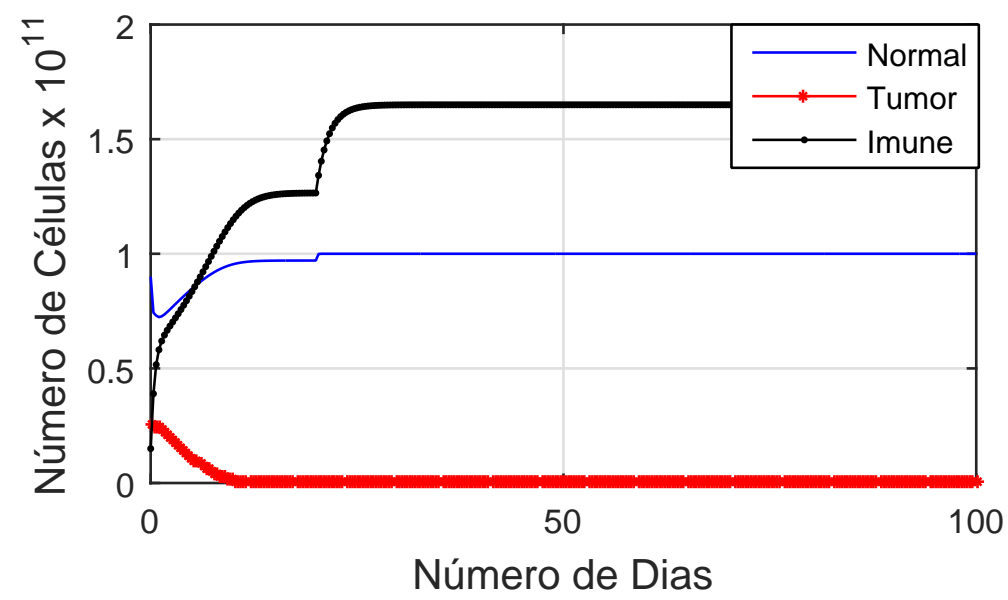

Figura 12: Simulação para o câncer de pele utilizando o fracionamento acelerado.

\begin{tabular}{ccccccc}
\hline $\begin{array}{c}\text { Tipo de } \\
\text { Fracionamento }\end{array}$ & $\begin{array}{c}\text { Tempo de } \\
\text { Eliminação do } \\
\text { do tumor }\end{array}$ & Dose/Fração & $\begin{array}{c}\text { Tempo de } \\
\text { Tratamento }\end{array}$ & $\begin{array}{c}\text { Dose } \\
\text { Total } \\
(\mathbf{G y})\end{array}$ & FN & FT \\
\hline Convencional & 45 dias & 30 frações de $2.0 \mathrm{~Gy}$ & 30 dias & 60.00 & 0.05 & 0.21 \\
Hipofracionamento & 40 dias & 20 frações de $3.0 \mathrm{~Gy}$ & 20 dias & 60.00 & 0.05 & 0.22 \\
\hline
\end{tabular}

mama é objeto de estudo de alguns autores, tais como, [11] [12] e [13]. Nesses estudos, grupos de pacientes foram submetidos a protocolos de tratamento com aplicações de quantidades de doses diferentes e os resultado obtidos com o uso do hipofracionamento mostrou-se satisfatório para o tratamento do câncer de mama. Os resultados das simulações encontram-se na Tabela 5.

De acordo com a Tabela 5, com o tratamento convencional o tumor é eliminado após 45 dias e 40 dias com o hipofracionamento. O tempo de eliminação do tumor utilizando esses dois fracionamentos estão próximos, assim, precisa-se analisar situações secundárias, como a exposição a radiação e o tamanho da dose utilizada. O paciente é curado em ambos os tratamentos. As Figuras 13 e 14 ilustram ambos os tratamentos.

\subsection{Discussão dos resultados}

Os resultados de simulações numéricas mostram que o fracionamento convencional, embora controle o tumor, talvez não seja a estratégia de tratamento ideal, pois resultados satisfatórios foram obtidos com outros regimes de tratamento. Como exemplo, pode-se citar o caso do câncer de pele, onde obteve-se resultados similares com uma significativa redução na dose total usando a estratégia de fracionamento acelerado.

Nos estudos realizados por [7], os resultados para os regimes hipofracionados no tratamento do câncer de próstata mostram um potencial de ganho terapêutico ao paciente. Nas simulações 


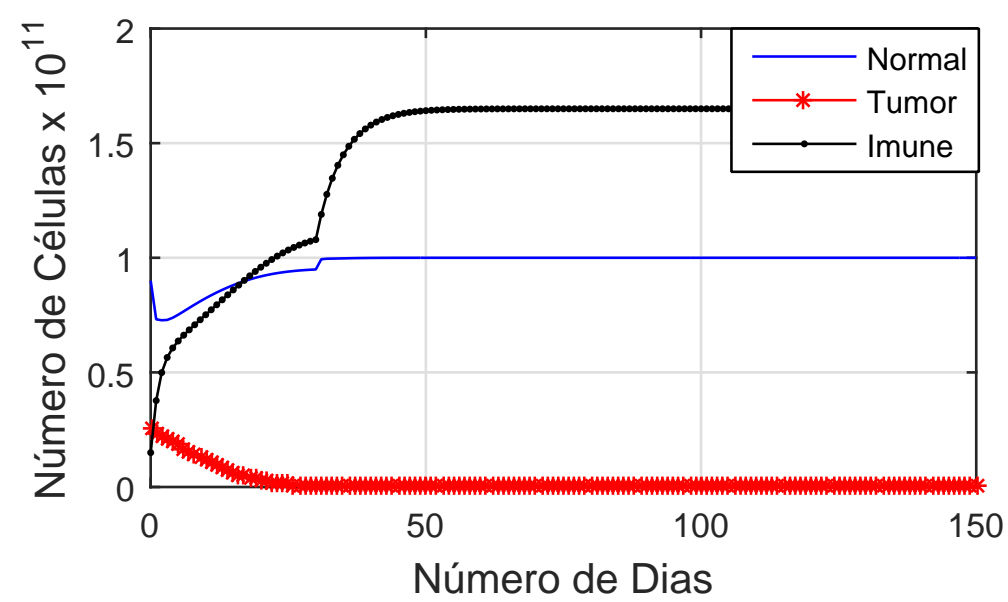

Figura 13: Simulações para o câncer de mama utilizando o tratamento convencional.

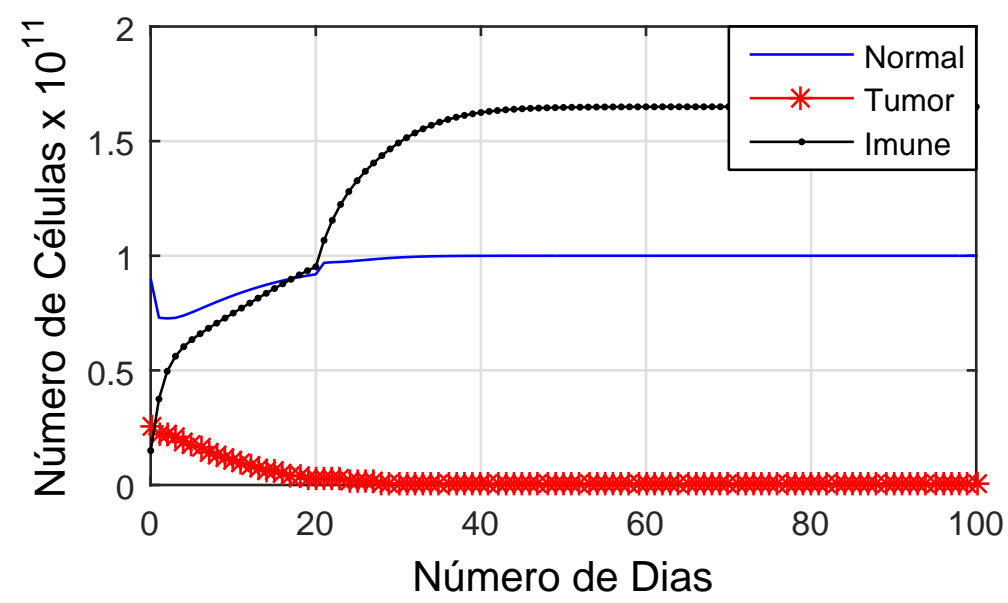

Figura 14: Simulações para o câncer de mama utilizando o hipofracionamento.

aqui realizadas, observou-se que usando a estratégia do hipofracionamento tem-se um ganho no tempo de tratamento e índices de controle similares, quando comparado com o fracionamento convencional. Portanto, os resultados numéricos obtidos vão ao encontro dos estudos realizados por [7] e podem contribuir para um tratamento menos agressivo aos pacientes.

E, ainda, várias estratégias de fracionamento não-convencional, testadas clinicamente, são apresentadas no trabalho de [5]. Os resultados por eles obtidos mostram que as estratégias de fracionamento não-convencional oferecem um ganho terapêutico promissor relacionado ao controle do tumor e nas complicações tardias aos pacientes, observadas nos tumores de cabeça e pescoço, câncer de pulmão e carcinoma de bexiga.

Portanto, existem diversos protocolos de fracionamento não-convencional que podem ser sugeridos para auxiliar no controle do tumor, a sua escolha depende de vários fatores, como por exemplo: o tipo de tumor, o estágio em que o tumor se encontra, sua agressividade, entre outros. Logo, as simulações numéricas de modelos tumorais combinados com um diagnóstico preciso da 
doença, realizado por especialistas da área, podem contribuir para a escolha do melhor protocolo de tratamento a ser adotado.

\section{Conclusões}

Neste trabalho foi estudado o modelo de crescimento tumoral proposto por [4]. A partir da análise de estabilidade foi possível analisar o comportamento do sistema na presença e na ausência do tumor, para que posteriormente fosse possível, através da aplicação da radioterapia, levar o sistema com tumor para o ponto de equilíbrio livre de tumor.

Nas simulações numéricas considerou-se o controle através do modelo linear quadrático, o qual descreve o tratamento por radioterapia, foram adotados critérios existentes na literatura e utilizados protocolos de tratamento diferentes para alguns estudos de casos. O objetivo foi analisar a dinâmica do crescimento do tumor através da aplicação de controle realizada por meio do modelo linear quadrático e verificar a veracidade dos protocolos padrões citados.

Considerando as modalidades de fracionamento utilizadas, observou-se que o uso de regimes não-convencionais de tratamento, ou seja, o hipofracionamento ou o fracionamento acelerado, mostraram resultados promissores, pois a partir das simulações realizadas verificou-se um ganho no tempo de eliminação de tumor e uma redução de dose. O fracionamento não convencional, portanto, apresenta-se como uma opção terapêutica importante para os casos de tumores analisados, tendo em vista a possibilidade de melhora do índice terapêutico com uma alteração na dose diária de radioterapia.

Vale destacar que a presente estimação dos parâmetros pode ser vista como uma diretriz para calcular a resposta do tumor e comparar a efetividade de estratégias de tratamento diferentes, embora qualquer novo esquema de tratamento deve ser corretamente testado em clínicas apropriadas.

Portanto, a partir da análise do modelo de crescimento tumoral foi possível analisar diversos protocolos de tratamento com o objetivo de controlar o câncer e minimizar os efeitos colaterais aos pacientes. Os resultados numéricos mostraram-se satisfatórios e a estratégia utilizada pode contribuir para a tomada de decisão de qual o melhor tratamento a ser considerado.

Assim, a modelagem matemática apresentada no presente trabalho pode ser considerada uma ferramenta útil, que tem o potencial para melhorar a qualidade do planejamento e tratamento do câncer, seja na economia de recursos ou na redução do tempo total de tratamento.

\section{Referências}

[1] Cancer Today. Disponível em: http://gco.iarc.fr/today Acesso em: 30 out. 2016.

[2] INCA. Instituto Nacional de Câncer José Alencar da Silva. O que é o câncer? Disponível em: http://www1.inca.gov.br/conteudo_view.asp?id=322. Acesso em: 31 out. 2015.

[3] STIEGELMEIER, E. W. Modelagem matemática do crescimento de tumores com tratamento através da radioterapia. 2007. 131 f. Dissertação (Mestrado em Modelagem Matemática) - Universidade Regional do Noroeste do Estado do Rio Grande do Sul, Ijuí, 2007. 
[4] DE PILLIS, L. G.; RADUNSKAYA, A. The dynamics of an optimally controlled tumor model: a case study. Mathematical and Computer Modelling, v. 37, n. 11, p. 1221-1244, 2003.

[5] MOONEM,L.; BARTELINK, H. Fractionation in radiotherapy. Cancer Treatment Reviews, v. 20, n. 4, p. 365-378, 1994.

[6] MU, X. et al. The effect of fraction time in intensity modulated radiotherapy: theoretical and experimental evaluation of an optimisation problem. Radiotherapy and Oncology, v. 68, n. 2, p. 181-187, 2003.

[7] FOWLER, J. F. et al. What hypofractionated protocols should be tested for prostate cancer? Radiation Oncology Biology Physics, v. 56, n. 4, p. 1093-1104, 2003.

[8] CLARK, J.; MCGEE, R. F. Enfermagem oncologica: um currículo básico. 2. ed. Porto Alegre: Editora Artes Médicas, 1997.

[9] CURY, F. L. B.; SOUHAMI, L. Hipofracionamento no câncer de próstata. Revista Brasileira de Cancerologia, v. 50, n. 3, p. 239-249, 2004.

[10] PELISSER, F. et al. Considerações sobre as modalidades radioterapêuticas empregadas para o tratamento de neoplasias na região de cabeça e pescoço. Revista da Faculdade de Odontologia-UPF, v. 13, n. 1, p. 75-79, 2008.

[11] SHELLEY, W. et al. A shorter fractionation schedule for postlumpectomy breast cancer patients. International Journal of Radiation Oncology Bioology Physics, v. 47, n. 5, p. 1219-1228, 2000.

[12] YARNOLD, J. et al. Fractionation sensitivity and dose response of late adverse effects in the breast after radiotherapy for early breast cancer: long-term results of a randomised trial. Radiotherapy and Oncology, v. 75, n. 1, p. 9-17, 2005.

[13] WHELAN, T. et al. Randomized trial of breast irradiation schedules after lumpectomy for women with lymph node-negative breast cancer. Journal of the National Cancer Institute, v. 94 , n. 15 , p. 1143-1150, 2002.

Artigo recebido em maio 2017 e aceito em ago. 2017. 